\title{
MR-guided high-intensity focused ultrasound treatment of uterine fibroids
}

REVIEW

This article was published in the following Dove Press journal:

Research and Reports in Focused Ultrasound

14 December 2016

Number of times this article has been viewed

\author{
Antonia Wiggermann' \\ Michael Beldoch' \\ Friederike Hoellen ${ }^{2}$ \\ Peter Hunold' \\ 'Clinic for Radiology and Nuclear \\ Medicine, ${ }^{2}$ Department of Obstetrics \\ and Gynecology, University Hospital \\ Schleswig-Holstein, Lübeck, Germany
}

Correspondence: Antonia Wiggermann Clinic for Radiology and Nuclear Medicine, University Hospital Schleswig-Holstein, Campus Lübeck, Ratzeburger Allee 160, Luebeck 23538, Germany Email Antonia.Wiggermann@uksh.de

\begin{abstract}
Uterine fibroids are benign, encapsulated uterine tumors, representing the most common reason for hysterectomy in premenopausal women. High-intensity focused ultrasound (HIFU; also known as focused ultrasound surgery) is a noninvasive technique leading to tissue heating in a centered focus, inducing a thermal tissue necrosis. Magnetic resonance-guided HIFU (MR-HIFU; also know as MR-guided focused ultrasound surgery) allows to allocate the uterine fibroids as well as to evaluate whether these are accessible for HIFU treatment. Further advantages are MR-based real-time temperature mapping and immediate evaluation of postprocedure therapeutic success by MR imaging. These advantages minimize the patient risk and make the treatment more effective. MR-HIFU is a uterus conserving, low pain-to-painless, and noninvasive treatment option. Here, we review its medical and economic advance to surgical procedures due to the low complication rates combined with proven symptom relief and the enhancement of the quality of life.
\end{abstract}

Keywords: high-intensity focused ultrasound, focused ultrasound surgery, uterine fibroids, real-time thermometry

\section{Introduction}

Uterine fibroids (UFs) are benign, encapsulated uterine tumors, representing the most common reason for hysterectomy in premenopausal women. ${ }^{1}$ As there is scarce incidence of UFs under the age of 25 years, there is an age-related cumulation with a prevalence of $20 \%$ at the age of 35 years and a growing prevalence up to $80 \%$ at the age of 50 years. ${ }^{2}$ Fifty-five percent of all UFs are located within the myometrium (intramural) or submucosal, whereas $40 \%$ of UFs are directly located below the uterine serosa. Although UFs are not associated with an increased mortality, UFs are frequently leading to a limited quality of life $(\mathrm{QoL})$ by various symptoms, such as dullness and vesicant dysmenorrhea, especially caused by intramural and subserosal UFs. Other symptoms are pollacisuria, constipation, sacral pain, and anemia due to the chronic prolonged blood loss. These symptoms are due to an expulsion phenomenon depending on the UF tumor size and localization. Intramural UFs are enucleated laparoscopically, UFs exceeding the size of $10 \mathrm{~cm}$ in diameter might be enucleated by laparotomy. Medical treatment options include progesterone-receptor-modulators which induce amenorrhea and shrinkage of UFs and show less side effects in comparison to GnRH analogues and proved to be more effective in comparison to gestagenbased contraceptives. ${ }^{3}$ Another less invasive treatment option is offered by uterine artery embolization (UAE). Here, the application of microspheres via endovascular 
catheterization occludes the UF vessel supply. ${ }^{4-6}$ Submucosal UFs are preferably treated by hysteroscopic resection. However, in women who have completed family planning, hysterectomy remains the most commonly used treatment of UFs worldwide. ${ }^{7,8}$ In women desiring pregnancy, laparoscopic enucleation of UF is the standard treatment. Especially in this case, uterine-conserving ablation technique is warranted. However until today, ablation techniques and embolization is not recommended in guidelines to women who wish to become pregnant, due to the hypothetical risk of myometrial deterioration and consecutive infertility or obstetrical complications. In 2004, the US Food and Drug Administration approved magnetic resonance-guided high-intensity focused ultrasound (MR-HIFU, synonym: MR-guided focused ultrasound surgery) as an entirely noninvasive approach in the treatment of UFs to reduce UFrelated symptoms and UF volume by thermal ablation. Due to its noninvasiveness, this technique is becoming more and more clinically relevant. ${ }^{9,10}$ It is a very low-pain to painless procedure with excellent therapeutic efficacy that could be consequently performed on an outpatient basis. ${ }^{11-15}$ As MRHIFU is an uterine-conserving treatment technique, it has a high acceptance in the group of women affected by UF. Due to its efficacy, MR-HIFU has emerged also as a treatment modality for prostate cancer and malignant liver tumors. ${ }^{16,17}$ For interventional radiologists, MR-HIFU is an attractive therapeutic option for UF and other pathologies.

\section{HIFU - application methods and physical mechanisms of tissue damage}

The main principle of HIFU is to deliver mechanical energy to the tissue by propagating through a solid or liquid medium. Contrary to diagnostic ultrasound probes that diverge ultrasound waves, HIFU probes are spherically curved to focus the ultrasound waves in a center. This means a 100-fold increase in mechanical (ultrasound) energy. The fundamental physical mechanism of HIFU is ultrasound absorption by tissues and its conversion into heat, leading to necrotic foci. These necrotic areas are defined as the so-called nonperfused volume (NPV). HIFU could be applied by sonographic guidance, as well as by magnetic resonance imaging (MRI) guidance. For sonographic-guided HIFU, penetration depth $>14 \mathrm{~cm}$ and NPV $>80 \%$ have been reported. ${ }^{18-20}$ The NPV as well as the tissue temperature distribution can be detected and quantified by contrast-enhanced MRI. Although the mechanisms are not yet totally understood, necrotic tissue destruction of UFs leads to volume shrinkage and a relief of various clinical symptoms. Reaching high NPVs is the mainstay predictor of therapeutical success in the MR-HIFU treatment of UFs. ${ }^{21-24}$ Another nonthermal tissue damaging effect of HIFU is caused by acoustic cavitation. This mechanical process occurs at high acoustic intensities and leads to the vaporization of tissue water and the formation of rapidly expanding microbubbles, which subsequently collapse with the release of shock waves and high-speed liquid jets, leading to tearing forces, which are followed by direct tissue damage. ${ }^{25-28}$ Thus, according to the recent US Food and Drug Administration safety criteria, safety margins of $1.5 \mathrm{~cm}$ between the treated UF volume and the adjacent peritoneal serosa have to be considered. ${ }^{24}$

\section{Inclusion criteria and limitations for MR-HIFU of UF}

UFs ranging from $2 \mathrm{~cm}$ to $>10 \mathrm{~cm}$ in size are basically suitable for MR-HIFU treatment. ${ }^{29}$ Scarring of the abdominal wall, eg, after cesarean section or laparatomy, might be the relative contraindications of MR-HIFU as far as it could not be bypassed by the ultrasound beam through dynamic focusing or movement of the HIFU probe. ${ }^{30}$ As there is the possibility of nerve damage, close allocation of the treated uterine fibroid next to the lumbar plexus should raise an enhanced level of attention. As the MR-HIFU beam is capable to propagate through tissues to a maximum depth of $14 \mathrm{~cm}$, UFs allocated deeper than $14 \mathrm{~cm}$ from the abdominal surface could not be reached. Thus, UFs of a retroflected uterus or in an adipose patient may not be accessible by MR-HIFU. In UFs allocated deeper than the maximum depth, filling the rectum with ultrasound gel might be an appropriate measure to reposition the uterus ventrally and mobilize the UF into the focus of the HIFU beam. As UFs can be relatively large tumors, intermittent cooling of the tissue could be necessary to prevent further damage. The relatively long treatment times of MR-HIFU could here mean a limitation in clinical practice. ${ }^{31}$

\section{HIFU therapy: treatment cells and temperature mapping}

Before executing HIFU treatment, a preparatory T2-weighted planning MRI in prone patient position is necessary to allocate the exact anatomical position and the tissue composition of the UF. Here, evaluation of the UF and target volume as well as of the accessibility for the HIFU beam is made. As the target volume is defined, it is then sonicated step by step as a cluster of so-called treatment cells of 4-16 $\mathrm{mm}$ in diameter (Figure 1). Due to the oscillatory movements of the ultrasound probe as well as using dynamic focusing of the HIFU beam in outwardly moving, concentric circles make volumetric sonication of 

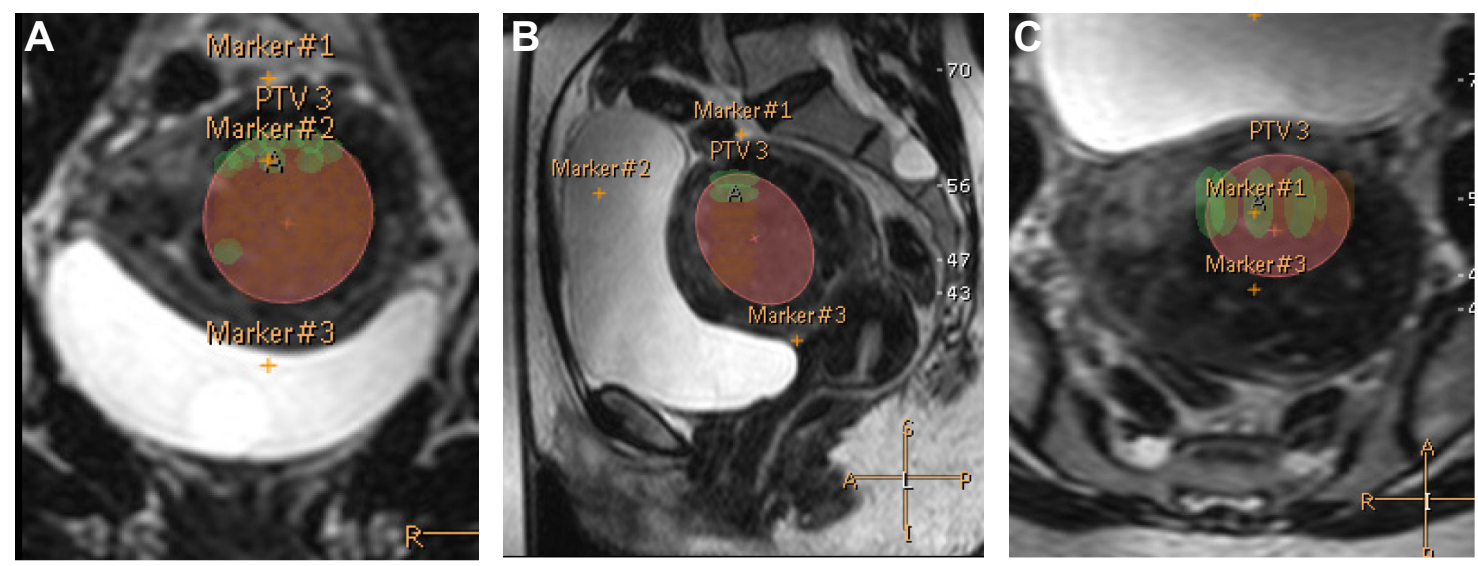

Figure I MR-HIFU treatment planning with superimposed MRI in three planes, such as coronal (A), sagittal (B), and transversal (C); pretreatment T2-weighted planning MR images show a uterine fibroid with low signal intensity. Red circles indicate the target volume for HIFU treatment as defined by the borders of the UF with a safety margin; green ellipsoids indicate the anticipated volumes of sonication.

Abbreviations: HIFU, high-intensity focused ultrasound; MR, magnetic resonance; MRI, magnetic resonance imaging; PTV, planned treatment volume.

these predetermined treatment cells feasible. Multiplanar real-time MR thermometry allows efficient monitoring of the ablation temperatures as well as structures at increased risk, especially in large volume UF ablations. ${ }^{32,33}$ Thus, energy of the HIFU beam can be adapted at any time during the ablation procedure ensuring an optimal tissue heating while minimizing the patient risk. This makes the treatment more effective and less time consuming. ${ }^{34,35}$

\section{Therapeutic outcome}

The most used parameter to evaluate the early therapeutic success is MRI evaluation of the UF necrosis immediately after the MR-HIFU procedure (Figure 2). Here, nonenhancing areas within the UF in contrast-enhanced T1-weighted images correspond with the NPV, which is the fundamental underlying correlation of midterm UF shrinkage. The so-called NPV ratio, determined as the NPV/UF volume, is the most reliable predicting factor for UF volume shrinkage. ${ }^{11,15,21-24}$ Using conventional MR-HIFU ablation technique without temperature mapping, very high NPV ratios up to $50 \%$ and in some cases $>90 \%$ are reported..$^{22,37-39}$ Here, it has to be critically mentioned that those very high NPV ratios might be due to the lower safety criteria leaving the patients at an increased risk of post-MR-HIFU complications. ${ }^{21,40}$ For clinical follow-up and assessment after MR-HIFU, UF symptom severity and health-related QoL questionnaires have been developed. Here, a significant increase in QoL 1 year after MR-HIFU is reported, even if the reduction of UF volume was only $25 \% .^{37,38,40,41}$

\section{Advantages for MR-HIFU treatment of UF}

As MR-HIFU is a noninvasive, uterus-conserving treatment method, it means an interesting treatment option especially for younger women still desiring pregnancy, pregnancy and healthy deliveries are possible after MR-HIFU treatment of UFs. ${ }^{42,43}$ Due to its noninvasiveness, MR-HIFU is significantly more cost effective, and thus, in economic advance compared to more invasive methods, such as UAE and hysterectomy. ${ }^{4}$ As surgical approaches are often accompanied
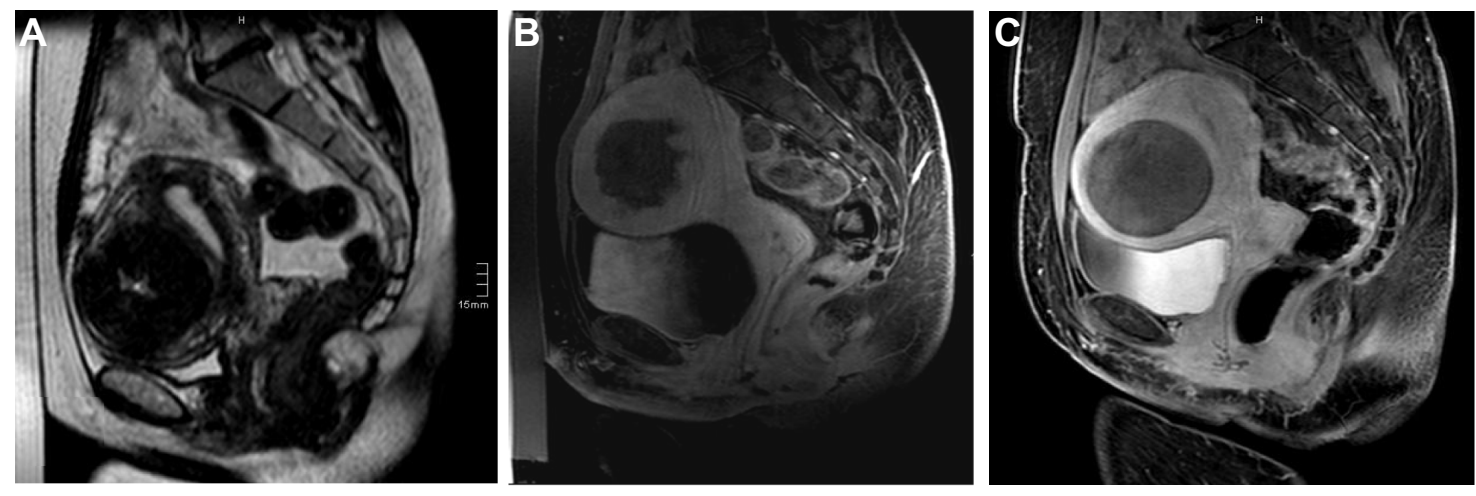

Figure 2 Sagittal T2-weighted MRI for planning purposes (A) shows a homogeneously hypointense UF in the anterior wall. Sagittal TI-weighted, fat-saturated and contrastenhanced MRI immediately (B) as well as I month (C) after the MR-HIFU procedure with a satisfying NPV.

Abbreviations: HIFU, high-intensity focused ultrasound; MR, magnetic resonance; MRI, magnetic resonance imaging; NPV, nonperfused volume; UF, uterine fibroid. 
by complications, such as intraoperative hemorrhage, injury of the uterus, and risk of emergency hysterectomy, MR-HIFU is an alternative low-risk treatment. ${ }^{44} \mathrm{MR}-\mathrm{HIFU}$ as well as UAE have lower complication rates and similar therapeutic success in terms of symptom and QoL improvement. ${ }^{45,46}$ Thus, MR-HIFU might mean a more complementary therapeutic approach in UF treatment in future. As targeted vessel ablation is feasible with MR-HIFU, this could be an interesting advancement of UF therapy combining the advantages of UAE and MR-HIFU: on demand, three-dimensional real-time reconstruction of the vessel supply on MRI followed by targeted MR-HIFU means a seminal approach in the therapy of UF and other pathologies..$^{31,32,47}$

\section{Complications and contraindications of MR-HIFU}

According to the German radiological-gynecological consensus meeting, MR-HIFU treatment of UFs is indicated in patients with UF-associated symptoms. Safe access for the HIFU beam is a prerequisite. In patients with an UF size varying between 3 and $10 \mathrm{~cm}$ and an UF number $<5$, MR-HIFU treatment might be indicated (Table 1). ${ }^{48}$ The complication rate of MR-HIFU therapy for UFs has been reported to be significantly lower than after surgical procedures. ${ }^{49}$ Pregnancy, suspicion of a uterine malignancy, and acute lower pelvic inflammations do mean an absolute contraindication for MR-HIFU therapy. Relative contraindications are UFs that might not be safely accessible by the ultrasound beam (due to huge abdominal wall scarring or interposed intestine), eg, patients with $>5$ UFs and UF size $>10 \mathrm{~cm}$ or $<3 \mathrm{~cm}$. Here, decision for HIFU therapy has to be made on a case-by-case basis (Table 2). ${ }^{30,48}$ Postinterventional abdominal discomfort, sciatic nerve paresthesia or simply leg pain, and slight skin burns of the abdominal wall are the commonly reported side effects of MR-HIFU treatment (Table 3). Only one case of severe skin burns that needed surgical intervention after MR-HIFU treatment is reported, further underlining the noninvasiveness of this method. ${ }^{31,48,50-54}$

Table I Indications for MR-HIFU therapy of UFs

UF-associated symptoms

Safe access window or the ultrasound beam to the UF

UF size $3-10 \mathrm{~cm}$

Number of UFs $<5$

Note: Data from Beck et al. ${ }^{48}$

Abbreviations: HIFU, high-intensity focused ultrasound; MR, magnetic resonance; UF, uterine fibroid.
Table 2 Contraindications for MR-HIFU treatment of UFs

Suspicion of an uterine malignancy (absolute)

Pregnancy (absolute)

Acute lower pelvic inflammation (absolute)

UF size $>10 \mathrm{~cm}$ (relative)

UF size $<3 \mathrm{~cm}$ (relative)

Number of UFs $>5$ (relative, decision on a case-by-case basis)

UFs not safely accessible by the ultrasound beam (eg, interposed

intestine, huge abdominal wall scarring, and dorsal allocation of the UF)

(relative)

Pedunculated subserosal UF (relative)

Close allocation of the UF to the os sacrum or UF allocated to the

posterior uterine wall (relative)

Contraindications for MRI contrast agents (relative)

General MRI contraindications

Note: Data from Beck et al. ${ }^{48}$

Abbreviations: HIFU, high-intensity focused ultrasound; MR, magnetic resonance; MRI, magnetic resonance imaging; UF, uterine fibroid.

\section{Outlook}

As new technical approaches offer new therapeutical perspectives, the application of HIFU is not limited to UF. MR-HIFU technique is an emerging treatment option in prostate and pancreatic cancers as well. Here, especially sonographic-guided HIFU applications have already been well established, whereas MR-HIFU approaches are now upcoming therapeutic advancements. ${ }^{16,55-57}$ Especially, targeted vessel ablation by MR-HIFU could mean an interesting approach in curative as well as palliative oncologic treatments. ${ }^{58} \mathrm{MR}-\mathrm{HIFU}$ treatment of breast cancer is now in development as tumor necrosis of up to $100 \%$ has been reported. ${ }^{59,60}$ Here, thermal-induced changes of vascular permeability for targeted drug delivery by HIFU might offer further interesting therapeutic opportunities.

\section{Conclusion}

MR-HIFU means a noninvasive, low pain-to-painless treatment option for UF, which has shown a significant improvement in QoL due to the reduction in UF symptoms.

Table 3 Adverse effects and complications after MR-HIFU therapy of UFs

Pain during the MR-HIFU treatment procedure (slight and only shorttermed in most cases)

(Slight) skin burns

(Slight) inflammation of the abdominal subepidermal fat and muscle tissue

Leg paresthesia due to nerve irritation or damage

Deep leg or pelvic vein thrombosis (very rare)

Intestinal perforation (extremely rare)

Note: Data from Beck et al. ${ }^{48}$

Abbreviations: HIFU, high-intensity focused ultrasound; MR, magnetic resonance; UF, uterine fibroid. 
As it is an uterus-conserving therapeutic approach, which further is in economic advance of surgical treatment procedures of UF, it should be offered especially to younger women. Relative contraindications for MR-HIFU of UF might be scarring of the abdominal wall, which could not be bypassed by the HIFU beam, as well as an extraordinary high body mass index.

\section{Disclosure}

Peter Hunold has received lecture fees from Philips Healthcare. The other authors report no conflicts of interest in this work.

\section{References}

1. Cramer SF, Patel A. The frequency of uterine leiomyomas. Am J Clin Pathol. 1990;94(4):435-438.

2. Baird DD, Dunson DB, Hill MC, Cousins D, Schectman JM. High cumulative incidence of uterine leiomyoma in black and white women: ultrasound evidence. Am J Obstet Gynecol. 2003;188(1): $100-107$.

3. Chavez NF, Stewart EA. Medical treatment of uterine fibroids. Clin Obstet Gynecol. 2001;44(2):372-384.

4. Zowall H, Cairns JA, Brewer C, Lamping DL, Gedroyc WM, Regan L. Cost-effectiveness of magnetic resonance-guided focused ultrasound surgery for treatment of uterine fibroids. BJOG. 2008;115(5): 653-662.

5. Rashid S, Khaund A, Murray LS, et al. The effects of uterine artery embolisation and surgical treatment on ovarian function in women with uterine fibroids. BJOG. 2010;117(8):985-989.

6. Banu NS, Gaze DC, Bruce H, Collinson PO, Belli AM, Manyonda IT. Markers of muscle ischemia, necrosis, and inflammation following uterine artery embolization in the treatment of symptomatic uterine fibroids. Am J Obstet Gynecol. 2007;196(3):213.e211-e215.

7. Stewart EA. Clinical practice. Uterine fibroids. $N$ Engl J Med. 2015;372(17):1646-1655.

8. Stewart EA. Uterine fibroids. Lancet. 2001;357(9252):293-298.

9. Ringold S. FDA approves ultrasound fibroid therapy. JAMA. 2004;292(23):2826.

10. Nieboer TE, Johnson N, Lethaby A, et al. Surgical approach to hysterectomy for benign gynaecological disease. Cochrane Database Syst Rev. 2009;(3):CD003677.

11. Tempany CM, Stewart EA, McDannold N, Quade BJ, Jolesz FA, Hynynen K. MR imaging-guided focused ultrasound surgery of uterine leiomyomas: a feasibility study. Radiology. 2003;226(3): 897-905.

12. Jolesz FA. MRI-guided focused ultrasound surgery. Annu Rev Med. 2009;60:417-430.

13. Funaki K, Fukunishi H, Funaki T, Kawakami C. Mid-term outcome of magnetic resonance-guided focused ultrasound surgery for uterine myomas: from six to twelve months after volume reduction. $J$ Minim Invasive Gynecol. 2007;14(5):616-621.

14. Kim YS, Rhim H, Choi MJ, Lim HK, Choi D. High-intensity focused ultrasound therapy: an overview for radiologists. Korean $J$ Radiol. 2008;9(4):291-302.

15. Stewart EA, Gostout B, Rabinovici J, Kim HS, Regan L, Tempany CM. Sustained relief of leiomyoma symptoms by using focused ultrasound surgery. Obstet Gynecol. 2007;110(2 pt 1):279-287.

16. Siddiqui K, Chopra R, Vedula S, et al. MRI-guided transurethral ultrasound therapy of the prostate gland using real-time thermal mapping: initial studies. Urology. 2010;76(6):1506-1511.

17. Zhang $\mathrm{L}$, Zhu H, Jin C, et al. High-intensity focused ultrasound (HIFU): effective and safe therapy for hepatocellular carcinoma adjacent to major hepatic veins. Eur Radiol. 2009;19(2):437-445.
18. Ren XL, Zhou XD, Yan RL, et al. Sonographically guided extracorporeal ablation of uterine fibroids with high-intensity focused ultrasound: midterm results. J Ultrasound Med. 2009;28(1):100-103.

19. Wang W, Wang Y, Wang T, Wang J, Wang L, Tang J. Safety and efficacy of US-guided high-intensity focused ultrasound for treatment of submucosal fibroids. Eur Radiol. 2012;22(11):2553-2558.

20. Leung JH, Yu SC, Cheung EC, et al. Safety and efficacy of sonographically guided high-intensity focused ultrasound for symptomatic uterine fibroids: preliminary study of a modified protocol. J Ultrasound Med. 2014;33(10):1811-1818.

21. Fennessy FM, Tempany CM, McDannold NJ, et al. Uterine leiomyomas: MR imaging-guided focused ultrasound surgery - results of different treatment protocols. Radiology. 2007;243(3):885-893.

22. Funaki K, Fukunishi H, Sawada K. Clinical outcomes of magnetic resonance-guided focused ultrasound surgery for uterine myomas: 24-month follow-up. Ultrasound Obstet Gynecol. 2009;34(5):584-589.

23. Lenard ZM, McDannold NJ, Fennessy FM, et al. Uterine leiomyomas: MR imaging-guided focused ultrasound surgery - imaging predictors of success. Radiology. 2008;249(1):187-194.

24. LeBlang SD, Hoctor K, Steinberg FL. Leiomyoma shrinkage after MRI-guided focused ultrasound treatment: report of 80 patients. AJR Am J Roentgenol. 2010;194(1):274-280.

25. Hill CR, Rivens I, Vaughan MG, ter Haar GR. Lesion development in focused ultrasound surgery: a general model. Ultrasound Med Biol. 1994;20(3):259-269.

26. Hynynen $\mathrm{K}$. The threshold for thermally significant cavitation in dog's thigh muscle in vivo. Ultrasound Med Biol. 1991;17(2):157-169.

27. Jenne JW, Preusser T, Gunther M. High-intensity focused ultrasound: principles, therapy guidance, simulations and applications. Z Med Phys. 2012;22(4):311-322.

28. Hesley GK, Gorny KR, Woodrum DA. MR-guided focused ultrasound for the treatment of uterine fibroids. Cardiovasc Intervent Radiol. 2013;36(1):5-13.

29. Venkatesan AM, Partanen A, Pulanic TK, et al. Magnetic resonance imaging-guided volumetric ablation of symptomatic leiomyomata: correlation of imaging with histology. J Vasc Interv Radiol. 2012; 23(6): 786-794.e784.

30. Okada A, Morita Y, Fukunishi H, Takeichi K, Murakami T. Non-invasive magnetic resonance-guided focused ultrasound treatment of uterine fibroids in a large Japanese population: impact of the learning curve on patient outcome. Ultrasound Obstet Gynecol. 2009;34(5):579-583.

31. Voogt MJ, Trillaud H, Kim YS, et al. Volumetric feedback ablation of uterine fibroids using magnetic resonance-guided high intensity focused ultrasound therapy. Eur Radiol. 2012;22(2):411-417.

32. Kohler MO, Mougenot C, Quesson B, et al. Volumetric HIFU ablation under 3D guidance of rapid MRI thermometry. Med Phys. 2009;36(8):3521-3535.

33. Chung AH, Jolesz FA, Hynynen K. Thermal dosimetry of a focused ultrasound beam in vivo by magnetic resonance imaging. Med Phys. 1999;26(9):2017-2026.

34. Gorny KR, Hangiandreou NJ, Hesley GK, Gostout BS, McGee KP, Felmlee JP. MR guided focused ultrasound: technical acceptance measures for a clinical system. Phys Med Biol. 2006;51(12):3155-3173.

35. Ruhnke H, Eckey T, Bohlmann MK, et al. MR-guided HIFU treatment of symptomatic uterine fibroids using novel feedback-regulated volumetric ablation: effectiveness and clinical practice. Rofo. 2013;185(10):983-991.

36. Eckey T, Neumann A, Bohlmann MK, Barkhausen J, Hunold P. Nichtinvasive thermoablation symptomatischer uterusmyome mit MR-gesteuertem hochenergetischen Ultraschall. [Non-invasive thermoablation of symptomatic uterine fibroids with magnetic resonance-guided highenergy ultrasound]. Radiologe. 2011;51:610-619. German.

37. de Melo FC, Diacoyannis L, Moll A, Tovar-Moll F. Reduction by $98 \%$ in uterine myoma volume associated with significant symptom relief after peripheral treatment with magnetic resonance imaging-guided focused ultrasound surgery. J Minim Invasive Gynecol. 2009;16(4):501-503. 
38. Dobrotwir A, Pun E. Clinical 24 month experience of the first MRgFUS unit for treatment of uterine fibroids in Australia. J Med Imaging Radiat Oncol. 2012;56(4):409-416.

39. Kim KA, Yoon SW, Yoon BS, Park CT, Kim SH, Lee JT. Spontaneous vaginal expulsion of uterine myoma after magnetic resonanceguided focused ultrasound surgery. J Minim Invasive Gynecol. 2011;18(1):131-134.

40. Hindley J, Gedroyc WM, Regan L, et al. MRI guidance of focused ultrasound therapy of uterine fibroids: early results. AJR Am J Roentgenol. 2004;183(6):1713-1719.

41. Yoon SW, Lee C, Kim KA, Kim SH. Contrast-enhanced dynamic MR imaging of uterine fibroids as a potential predictor of patient eligibility for MR guided focused ultrasound (MRgFUS) treatment for symptomatic uterine fibroids. Obstet Gynecol Int. 2010;2010:834275.

42. Rabinovici J, David M, Fukunishi H, et al; MRgFUS Study Group. Pregnancy outcome after magnetic resonance-guided focused ultrasound surgery (MRgFUS) for conservative treatment of uterine fibroids. Fertil Steril. 2010;93(1):199-209.

43. Zaher S, Lyons D, Regan L. Uncomplicated term vaginal delivery following magnetic resonance-guided focused ultrasound surgery for uterine fibroids. Biomed Imaging Interv J. 2010;6(2):e28.

44. Griffiths A, D’Angelo A, Amso N. Surgical treatment of fibroids for subfertility. Cochrane Database Syst Rev. 2006;(3):CD003857.

45. van der Kooij SM, Hehenkamp WJ, Volkers NA, Birnie E, Ankum WM, Reekers JA. Uterine artery embolization vs hysterectomy in the treatment of symptomatic uterine fibroids: 5-year outcome from the randomized EMMY trial. Am J Obstet Gynecol. 2010;203(2):105. e101-e113.

46. Bratby MJ, Belli AM. Radiological treatment of symptomatic uterine fibroids. Best Pract Res Clin Obstet Gynaecol. 2008;22(4):717-734.

47. Wu F, Chen WZ, Bai J, et al. Tumor vessel destruction resulting from high-intensity focused ultrasound in patients with solid malignancies. Ultrasound Med Biol. 2002;28(4):535-542.

48. Beck A, David M, Kröncke T. Magnetresonanz-geführter fokussierter Ultraschall zur Myombehandlung - Ergebnisse des ersten radiologisch - gynäkologischen Expertentreffens. [MR guided treatment of uterine fibroids with high focused ultrasound - results of the first radiologicgynecologic expert meeting]. Fortschr Röntgenst. 2013;185:464-466. German.

49. Taran FA, Tempany CM, Regan L, et al; MRgFUS Group. Magnetic resonance-guided focused ultrasound (MRgFUS) compared with abdominal hysterectomy for treatment of uterine leiomyomas. Ultrasound Obstet Gynecol. 2009;34(5):572-578.
50. Leon-Villapalos J, Kaniorou-Larai M, Dziewulski P. Full thickness abdominal burn following magnetic resonance guided focused ultrasound therapy. Burns. 2005;31(8):1054-1055.

51. Machtinger R, Inbar Y, Cohen-Eylon S, Admon D, Alagem-Mizrachi A, Rabinovici J. MR-guided focus ultrasound (MRgFUS) for symptomatic uterine fibroids: predictors of treatment success. Hum Reprod. 2012;27(12):3425-3431.

52. Zhang L, Chen WZ, Liu YJ, et al. Feasibility of magnetic resonance imaging-guided high intensity focused ultrasound therapy for ablating uterine fibroids in patients with bowel lies anterior to uterus. Eur $J$ Radiol. 2010;73(2):396-403

53. Morita Y, Ito N, Hikida H, Takeuchi S, Nakamura K, Ohashi H. Noninvasive magnetic resonance imaging-guided focused ultrasound treatment for uterine fibroids - early experience. Eur J Obstet Gynecol Reprod Biol. 2008;139(2):199-203.

54. Desai SB, Patil AA, Nikam R, Desai AS, Bachhav V. Magnetic resonance-guided focused ultrasound treatment for uterine fibroids: first study in Indian women. J Clin Imaging Sci. 2012;2:74.

55. Feijoo ER, Sivaraman A, Barret E, et al. Focal high-intensity focused ultrasound targeted hemiablation for unilateral prostate cancer: a prospective evaluation of oncologic and functional outcomes. Eur Urol. 2016;69(2):214-220.

56. Khokhlova TD, Hwang JH. HIFU for palliative treatment of pancreatic cancer. J Gastrointest Oncol. 2011;2(3):175-184.

57. Anzidei M, Marincola BC, Bezzi M, et al. Magnetic resonance-guided high-intensity focused ultrasound treatment of locally advanced pancreatic adenocarcinoma: preliminary experience for pain palliation and local tumor control. Invest Radiol. 2014;49(12):759-765.

58. Hynynen K, Colucci V, Chung A, Jolesz F. Noninvasive arterial occlusion using MRI-guided focused ultrasound. Ultrasound Med Biol. 1996;22(8):1071-1077.

59. Zippel DB, Papa MZ. The use of MR imaging guided focused ultrasound in breast cancer patients; a preliminary phase one study and review. Breast Cancer. 2005;12(1):32-38.

60. Deckers R, Merckel LG, Denis de Senneville B, et al. Performance analysis of a dedicated breast MR-HIFU system for tumor ablation in breast cancer patients. Phys Med Biol. 2015;60(14):5527-5542.
Research and Reports in Focused Ultrasound

\section{Publish your work in this journal}

Research and Reports in Focused Ultrasound is an international, peerreviewed, open access online journal publishing original research, study protocols, reports, editorials, reviews and commentaries on all aspects of focused ultrasound technologies for the treatment of cancer and other diseases. The manuscript management system is completely

\section{Dovepress}

online and includes a very quick and fair peer-review system, which is all easy to use. Visit http://www.dovepress.com/testimonials.php to read real quotes from published authors. 